\title{
Role of autophagy in resistance to oxaliplatin in hepatocellular carcinoma cells
}

\author{
HAILEI DU ${ }^{1}$, WEIPING YANG ${ }^{1}$, LIN CHEN $^{1}$, MINGMING SHI ${ }^{2}$, VARUN SEEWOO ${ }^{1}$, \\ JIAYU WANG ${ }^{1}$, ANDY LIN ${ }^{1}$, ZHUORAN LIU ${ }^{1}$ and WEIHUA QIU ${ }^{1}$ \\ ${ }^{1}$ Department of Surgery, Ruijin Hospital, and ${ }^{2}$ Institute of Digestive Surgery, \\ Shanghai Jiao Tong University School of Medicine, Shanghai 200025, P.R. China
}

Received July 9, 2011; Accepted August 30, 2011

DOI: $10.3892 /$ or.2011.1464

\begin{abstract}
In spite of an initially promising anti-tumor activity, oxaliplatin-based combinatorial treatments can eventually result in a tumor resistance response. In this study we aimed to understand the role of autophagy in HCC cell resistance to oxaliplatin and to discuss its potential therapeutic implication. We found that exposure to oxaliplatin induced a significant increase in LC3 lipidation and subsequent LC3 puncta formation. While the proliferation of HCC cells was inhibited upon oxaliplatin exposure, inhibition of autophagy by ATG7 interference and chloroquine pre-treatment further increased the sensitivity to chemotherapy. Meanwhile, the oxaliplatininduced apoptotic cell death was significantly enhanced. These results suggest that autophagy may function importantly in HepG2 cell resistance to oxaliplatin. Intriguingly, the resistance could be recovered apparently by inhibition of autophagy. This also points to the potential therapy for hepatoma by perturbing autophagy.
\end{abstract}

\section{Introduction}

Hepatocellular carcinoma (HCC) is one of the most common cancers in many parts of the world, particularly in the Far East, South Sahara and Southern Europe (1). Over recent years, the occurrence of HCC has actually increased markedly in lowrisk countries such as the US (2). HCC is often diagnosed at an advanced stage when most potentially curative therapies such

Correspondence to: Dr Weihua Qiu, Department of Surgery, Ruijin Hospital, Shanghai Jiao Tong University School of Medicine, 197 Ruijin Er Road, Shanghai 200025, P.R. China

E-mail: drqwh2003@hotmail.com

Abbreviations: ATG7, autophagy-related protein 7; LC3, microtubule-associated protein 1 light chain 3; p62, sequestosome 1; OX, oxaliplatin; CQ, chloroquine; CCK8, cell counting kit-8; MDC, monodansylcadaverine; HCC, hepatocellular carcinoma

Key words: hepatocellular carcinoma, ATG7, oxaliplatin, autophagy, apoptosis as resection, transplantation or percutaneous and transarterial interventions are of limited efficacy $(2,3)$. Although sorafenib has shown optimal results in the improvement of survival and the time to radiological progression in patients with advanced $\mathrm{HCC}$, the mechanism of chemotherapy resistance and new therapeutic strategies still need to be explored (5).

Oxaliplatin, a diaminocyclohexane-containing platinum, has been widely used in the treatment of a variety of human tumors and has shown promising results in clinical applications. In addition, oxaliplatin has fewer side effects compared to other platinum drugs in terms of nephrotoxicity and myelosuppression (6). Recently, oxaliplatin-based combinatorial treatments have shown a promising anti-tumor activity in patients with HCC (7). However, as other forms of chemotherapy, oxaliplatin treatment can eventually result in tumor resistance in spite of the initial response. Multiple mechanisms related to the resistance to platinum drug have been extensively studied, which include drug accumulation deficiencies, intracellular detoxification by conjugation with glutathione, reduced DNA-platinum adduct formation, alterations in transport proteins and downstream signaling affecting cell death pathways. However, the chemo-resistance to oxaliplatin in HCC has not been completely understood.

Autophagy is a dynamic process involving the bulk degradation of cytoplasmic organelles and proteins. Based on the function of cellular recycling, autophagy plays key roles in the quality control of cellular components as well as supplying nutrients and materials for newly constructed structures in cells under metabolic stresses (8). Recent studies suggest that autophagy may be important not only in the regulation of cancer development and progression, but also in determining the response of tumor cells to anticancer therapy $(9,10)$. It has also been observed that increased autophagy is closely associated with chemotherapy, which may cause autophagic tumor cell death or adaptation to drug cytotoxicity. Considering the paradoxical function of autophagy, autophagic cell death, and apoptosis in cancer, the exact role and the relationship of autophagy and chemotherapeutic resistance are still poorly understood and need further elucidated. Thus, illuminating the role of autophagy and its underlying mechanisms in enhancing cell survival upon chemotherapy and sensitizing cell death by autophagy targeting would have a significant impact on the treatment of HCC. 
In the present study, we investigated the induction of autophagy by oxaliplatin in HCC HepG2 cells and its role in chemoresistance. We found that oxaliplatin significantly induced autophagy, and inhibition of autophagy sensitized cells to chemotherapy. These results suggest that targeting autophagy may abrogate the resistance of HCC cells to oxaliplatin and can lead to the development of novel therapeutic approaches.

\section{Materials and methods}

Cell culture, treatment and RNA extractions. The HCC cell line HepG2 was purchased from American Type Culture Collection (Rockville, MD), and cultured as previously described (11). Cells were plated $24 \mathrm{~h}$ before treatment and were at subconfluent density at the time of drug exposure. Oxaliplatin (L-OHP; Boryung Pharmaceutical, Seoul, Korea), a diaminocyclohexane-containing platinum, was dissolved in dimethylsulfoxide (DMSO) and diluted with DMEM to achieve a desired concentration of $0,8,16,32,64,128$, and $256 \mu \mathrm{M}$. Forty-eight hours after treatment, media were removed and cells were washed with PBS. In the inhibitory study of autophagic-lysosomal protein degradation, cells were pre-treated with chloroquine (CQ) (Sigma, St. Louis, MO) from 125, 250, 500 to $1000 \mu \mathrm{M}$ for $12 \mathrm{~h}$, followed by oxaliplatin for another $48 \mathrm{~h}$. Total RNA was isolated using the RNeasy Mini kit (Qiagen, Valencia, CA). RNA quality was tested by running on a $1.2 \%$ DEPC/MOPS agarose gel, and the concentration was measured by UV spectroscopy. RNA was stored in DEPC water with $10 \mathrm{mM}$ DTT and RNasin $(1 \mathrm{U} / \mathrm{ml})$ at $-70^{\circ} \mathrm{C}$.

Colorimetric viability assay. Cell viability was estimated using the colorimetric assay. HepG2 cells were plated, in triplicate, at a density of 5,000 cells/well in a volume of $100 \mu \mathrm{l}$ in 96-well microtiter plates and incubated overnight. On the following day, oxaliplatin was added to the wells at the dosage mentioned above. Plates were incubated for an additional $48 \mathrm{~h}$. Then, $20 \mu \mathrm{l}$ of the cell-counting solution (CCK8; Dojindo Laboratories, Japan) was added to each well, and incubation was carried out at $37^{\circ} \mathrm{C}$ for $3 \mathrm{~h}$. The absorbance of the solution was read spectrophotometrically at $450 \mathrm{~nm}$ with a reference at $650 \mathrm{~nm}$ using a microtiter plate reader (Becton-Dickinson). Cell viability was calculated according to the following formula: Cell viability $(\%)=\left[1-\left(\mathrm{A} 450_{\text {sample }}-\mathrm{A} 450_{\text {blank }}\right) /(\mathrm{A} 450\right.$ control $\left.-\mathrm{A} 450_{\text {blank }}\right)$ ] x 100 . The $\mathrm{IC}_{50}$ value, at which $50 \%$ of the cell growth inhibition compared with DMSO control, was determined by constructing a dose-response curve and calculated at Website: $\mathrm{ED}_{50}$ and $\mathrm{IC}_{50}$ for Graded Response (Ver 1.2, http://chiryo.phar.nagoya-cu.ac.jp/javastat/Graded50-j.htm).

Detection of acidic vesicular organelles (AVOs) with MDC staining. Autophagy is characterized by acidic vesicular organelle (AVO) formation, and the living cell special staining with MDC (monodansylcadaverine; Sigma, St. Louis, MO) was performed to morphologically detect AVOs in HepG2 cells after oxaliplatin treatment. The cells were incubated with $\mathrm{MDC}$ at a final concentration of $0.05 \mathrm{mM}$ away from light at $37^{\circ} \mathrm{C}$ for $60 \mathrm{~min}$ and collected in $10 \mathrm{mM}$ Tris- $\mathrm{HCl}$, pH 8.0 containing $0.1 \%$ Triton X-100. After washing 2 times in PBS, the fluorescence of MDC was measured at an excitation wavelength $380 \mathrm{~nm}$ and an emission wavelength of $530 \mathrm{~nm}$ under an epifluorescence microscope (Olympics IX71). A typical MDC accumulation in AVOs exhibited distinct dotlike structures distributed within the cytoplasm or localized in the perinuclear regions, indicating autophagosome formation. The quantitation of intracellular MDC fluorescence intensity was measured by flow cytometry.

Observation of immunofluorescence for LC3-positive autophagic vacuoles. Forty-eight hours after oxaliplatin treatment, HepG 2 cells were fixed with $4 \%$ paraformaldehyde for $30 \mathrm{~min}$ and made permeable with methanol at $-20^{\circ} \mathrm{C}$ for $10 \mathrm{~min}$. Cells were blocked using $10 \%$ goat serum at room temperature for $30 \mathrm{~min}$. Then, cells were incubated with rabbit polyclonal antiLC3 and mouse monoclonal anti-p62 SQSTM1 antibodies (Santa Cruz Biotechnology, Santa Cruz, CA). Goat anti-rabbit IgG-FITC and anti-mouse IgG-TR secondary antibodies (Santa Cruze Biotechnology) were employed for fluorescent coloration. Immunofluorescent images were captured using an epifluorescence microscope (Olympics IX71).

Quantitative real-time PCR. Since endogenous levels of microtubule-associated protein light chain 3 (LC3) is a specific marker of autophagosomes in mammalian cells, it is now widely used for autophagy measurement (12). Changes in LC3 mRNA were further detected quantitatively by real-time PCR using Premix Ex Taq (Takara, Japan). Total RNA was extracted from the cells using TRIzol reagent (Invitrogen) and treated with RNA-free DNase I (Promega, Madison, WI). Total RNA was reversely transcribed to cDNA using RevertAid firststrand cDNA synthesis kit (Fermentas, Canada). GAPDH was used as an endogenous control to normalize for differences in the amount of total RNA in each sample. Primers for $L C 3$ and $G A P D H$ were as follows: $L C 3,5$ '-TAC GAG CAG GAG AAA GAC GAG G-3' and 5'-GGC AGA GTA GGT GGG TTG GTG-3'; GAPDH, 5'-GAAGGTGAAGGTCGGAGTC-3' and 5'-GAAGATGGTGATGGGATTTC-3'. Thermal cycling was performed in a 7000 Sequence Detection System (Applied Biosystems). Reactions were carried out in a $20-\mu 1$ reaction volume. PCR amplification conditions were as follows: $95^{\circ} \mathrm{C}$ for $10 \mathrm{~min}, 40$ cycles of $95^{\circ} \mathrm{C}$ for $5 \mathrm{sec}$, and $60^{\circ} \mathrm{C}$ for $30 \mathrm{sec}$. The $\mathrm{Ct}$ data were obtained automatically, and each data point was performed in triplicates.

Inhibition of autophagy by RNA interference. As an essential autophagy gene, ATG7 is an E1-like enzyme involved in the ubiquitination of MAP-LC3 early in the pathway, (13) and knockdown of ATG7 inhibits autophagy specifically (14). Therefore, HepG2 cells were pre-treated with $A T G 7$ interference to assess the contribution of autophagy in promoting HCC cell resistance to oxaliplatin. The ATG7-targeting sense sequence and the universal negative control siRNA were purchased from Invitrogen (12935-400). The human ATG7 sequence (5'-GGAGTCACAGCTCTTCCTT-3') was cloned into BamH1 and EcoR1 sites of the pGSU6-GFP vector (Genlantis, GTP600300). Control scrambled and shRNA plasmids were transfected into HepG2 cells plated at $50 \%$ confluence in 6-well plates using Lipofectamine 2000 (Invitrogen, 11668019). Forty-eight hours after transfection, cells were washed with PBS and collected for flow cytometry. 
Then, the cells with ATG7 siRNA and control siRNA were treated with oxaliplatin as mentioned above.

Apoptosis assay. The apoptotic status of HepG2 cells was evaluated by measuring the exposure of phosphatidylserine on the cell membranes using Annexin V-fluorescein isothiocyanate (Annexin V-FITC) and propidium iodide (PI) staining. The BD Pharmingen Annexin V-FITC Apoptosis Detection Kit I (BD Biosciences, Franklin Lakes, NJ, USA) was used for the apoptosis assay. Forty-eight hours after oxaliplatin treatment, the HepG2 cell pellets were centrifuged and washed twice with cold PBS and suspended in 100- $\mu$ l binding buffer. The cells were incubated with $5 \mu \mathrm{l}$ Annexin V-FITC and $5 \mu \mathrm{l}$ $\mathrm{PI}$ at room temperature for $15 \mathrm{~min}$ in the dark. An additional $400 \mu 1$ of $1 \mathrm{X}$ binding buffer was added to each tube. Samples were analyzed immediately by flow cytometry (FACScan, Becton-Dickinson, USA). One thousand events were acquired using green channel FL1 for Annexin V-FITC and the red channel FL3 for PI. Annexin $\mathrm{V}^{+} / \mathrm{PI}^{-}$cells were considered early apoptotic, and Annexin $\mathrm{V}^{+} / \mathrm{PI}^{+}$cells were considered late apoptotic and necrotic. In the present study, both subpopulations were counted together and expressed as the total fraction of apoptotic cells.

Isolation of proteins and immunoblot analyses. Cells were harvested at various time points by centrifugation at $800 \mathrm{rpm}$ for $5 \mathrm{~min}$ at $4^{\circ} \mathrm{C}$. The isolation of proteins, electrophoresis, and the polyvinylidene difluoride membrane transfer were performed as previously described (15). Blots were probed with polyclonal rabbit anti-LC3, monoclonal mouse anti-caspase-3, mouse monoclonal anti-p62 and GAPDH polyclonal antibodies (Santa Cruz Biotechnology) overnight at $4^{\circ} \mathrm{C}$. GAPDH was used as an internal control. Goat anti-rabbit or goat antimouse IgG secondary antibody (Santa Cruz Biotechnology) was incubated with the polyvinylidene difluoride membranes at room temperature for $1 \mathrm{~h}$ and then washed with $0.5 \%$ I-block blocking buffer. The blots were incubated with chemiluminescent substrate and detected by enhanced chemiluminescence assay (Millipore). Band density was measured by densitometry, quantitated using Photoshop software (Adobe, San Jose, USA), and normalized to an indicated sample in the identical membrane. Blots shown are representative of at least three independent experiments.

Statistical analysis. Statistical analysis was performed using SPSS software (v.13.0). Data are expressed as mean \pm SD of at least three independent experiments. Comparisons of quantitative data were analyzed using the Student's t-test between two groups or by one-way ANOVA for multiple groups. A statistically significant difference was defined as a $\mathrm{p}$-value $<0.05$.

\section{Results}

Effect of oxaliplatin and chloroquine on cell viability. The cell viability assay was used to evaluate cytotoxic effect of oxaliplatin on HepG2 cells. As shown in Fig. 1, oxaliplatin (0-256 $\mu \mathrm{mol} / \mathrm{l})$ produced a dose- and time-dependent reduction in HepG2 growth. The $\mathrm{IC}_{50}$ for $48 \mathrm{~h}$ of oxaliplatin treatment in HepG2 cells was $12.10 \pm 5.03 \mu \mathrm{M}$. It reached $\sim 55 \%$ with $18 \mu \mathrm{mol} / \mathrm{l}$ oxaliplatin treatment for $48 \mathrm{~h}$. Therefore, we used

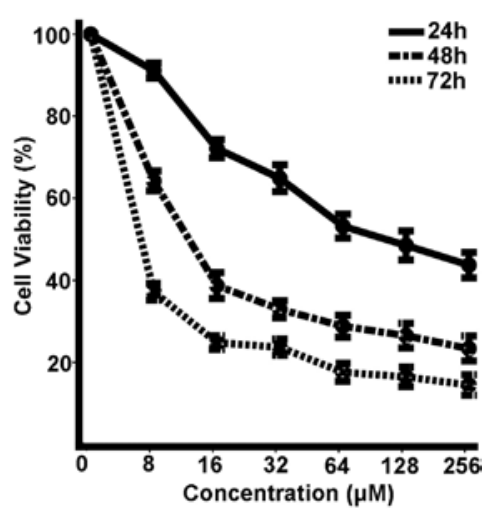

Figure 1. Effects of oxaliplatin on cell viability in HepG2 cells. HepG2 cells were incubated with different concentrations $(0-256 \mu \mathrm{M})$ of oxaliplatin for 24, 48 and $72 \mathrm{~h}$. Cell viability was measured with an MTT assay. $\mathrm{IC}_{50}$ was calculated for the further experiments. All data are representative of three independent experiments.

$18 \mu \mathrm{M}$ of oxaliplatin for $48 \mathrm{~h}$ in the HepG2 cells for further experiments. The viability assay was also employed to identify the dosage of chloroquine (CQ) for the autophagic proteolysis inhibition study. The HepG2 viability decreased to $91.70 \pm 12.03$ and $89.64 \pm 13.13 \%$ for cells treated with 125 and $250 \mu \mathrm{M} \mathrm{CQ}$. The viability further decreased to $84.78 \pm 13.16$ and $83.49 \pm 14.72 \%$ at the dosage of 500 and $1000 \mu \mathrm{M} \mathrm{CQ}$, respectively. The dose of CQ was set at $80 \mu \mathrm{M}$ since it was the dose that mostly induced AVO formation, the marker of autophagy, with a minimal inhibitory effect on the HepG2 cells.

Confirmation of ATG7 knockdown by siRNA. After transfection of the HepG2 cells with the ATG7-targeting siRNA and control siRNA plasmids, $A T G 7$ knockdown was confirmed using immunoblotting. As showed in Fig. 2a, inhibition of $A T G 7$ protein was marked, and the endogenous expression was only $\sim 56 \%$ of the level in the mock study (control siRNA transfection).

Induction of autophagy by oxaliplatin in HepG2 cells. Observation of the immunofluorescence for LC3-positive AVO formation was initially employed to detect the induction of autophagy by oxaliplatin in the HepG 2 cells. As shown in Fig. 2b, living cell staining with MDC revealed a significant increase in punctas formation after oxaliplatin treatment with control siRNA pre-transfection (Ox+Control siRNA group), which was mainly localized in the perinuclear regions. In contrast, there was only a slight increase in AVO formation in the cytoplasm of cells with ATG7 siRNA pre-transfection even when followed by oxaliplatin treatment $(\mathrm{Ox}+\mathrm{ATG} 7$ siRNA group). In the mock study, without oxaliplatin, few AVOs were noted in the cells with control siRNA and ATG7 siRNA transfection.

Findings of the dual immunofluorescence for LC3 and p62 revealed similar results as the living cell staining by MDC. A significant increase in the red-to-green fluorescence ratio (AVO accumulation within the cytoplasm) was noted in response to oxaliplatin treatment in HepG2 cells $(\mathrm{Ox}+\mathrm{Control}$ siRNA group) (Fig. 2c). More importantly, this increase was abolished by ATG7 knockdown (Ox+ATG7 siRNA group). 

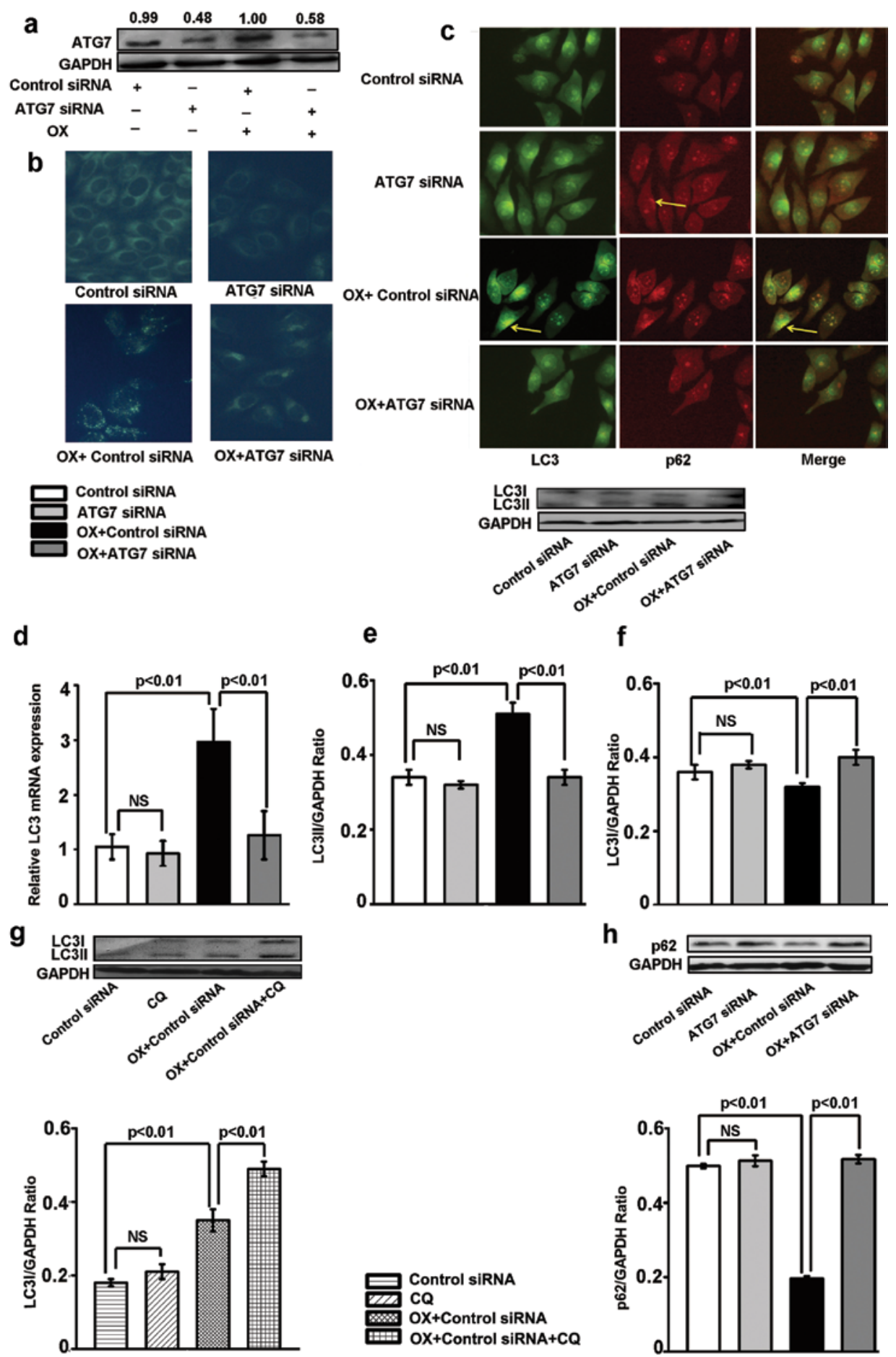

Figure 2. The role of autophagy in the cell response to oxaliplatin. (a) HepG2 cells were transfected with an ATG7 shRNA or control RNA plasmid using the pGSPU6 expression vector and a Lipofectamine transfection protocol. Western blots of ATG7 expression in HepG2 cells are shown. (b) Autophagic vacuoles were observed with MDC staining. (c) Finding of the immunofluorescence for LC3-positive and p62-positive autophagic vacuoles. (d) LC3 mRNA levels were measured by quantitative real-time RT-PCR analyses. Changes in LC3 lipidation and LC3-I expression were monitored by immunoblotting with ATG7 interference (e and f), and with CQ treatment (g). (h) Following oxaliplatin treatment, p62 accumulation with or without $A T G 7$ interference was detected by immunoblotting as well. The data shown here are representative of three independent experiments. Densitometry was performed using Photoshop, and the results are presented as mean $\pm \mathrm{SD}$.

Quantitative real-time PCR analysis showed the mean ratios of $L C 3$ to $G A P D H$ mRNA increased significantly to $2.97 \pm 0.60$ from $1.05 \pm 0.23$ in the control group $(\mathrm{p}<0.01)$ following oxaliplatin treatment (Fig. 2d). Steady-state $L C 3$ mRNA was identified in the HepG2 cells with ATG7 siRNA pre-transfection and oxaliplatin treatment in the mock study.
Western blot analysis of LC3 lipidation (LC3-II) is regarded as a reliable means to monitor autophagy (16). Quantitative analyses in Fig. 2e revealed that the post-oxaliplatin LC3 lipidation increased markedly from $0.34 \pm 0.03$ to $0.51 \pm 0.03$ $(\mathrm{p}<0.01)$ after normalization. In addition, pre-treatment of $A T G 7$ interference inhibited the increase in LC3 lipida- 

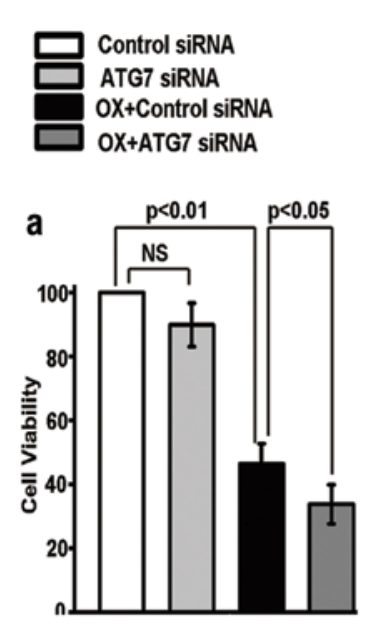

C
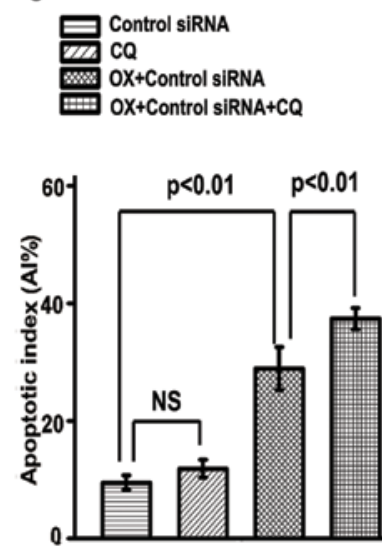

b
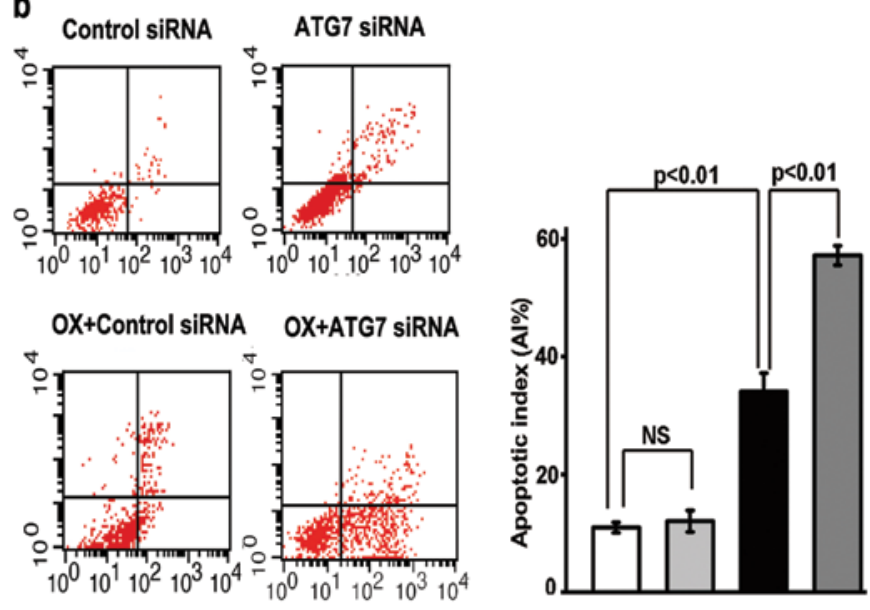

d Caspase-3
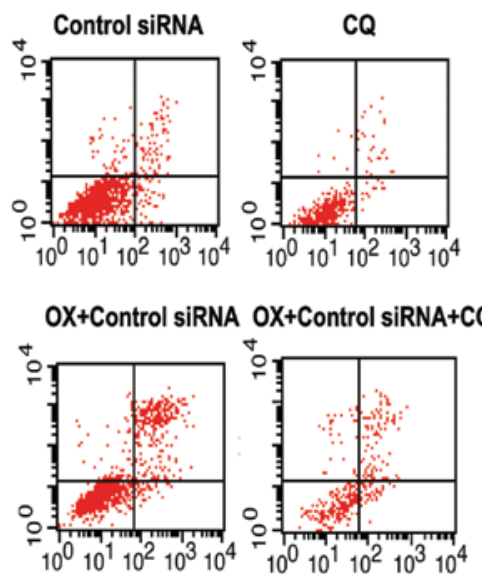
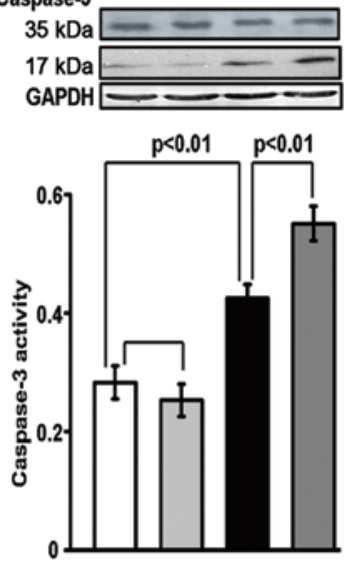

Figure 3. Relationship between inhibition of autophagy and apoptotic pathway activation by oxaliplatin. Cells were divided into four groups: Control siRNA, ATG7 siRNA, oxaliplatin plus control siRNA and oxaliplatin plus ATG7 siRNA. (a) Cell proliferation was determined by CCK8 assays. (b and c) Autophagy inhibition by $A T G 7$ interference and CQ treatment enhanced the oxaliplatin-induced apoptotic cell death as determined by flow cytometry after Annexin V and PI staining. (d) Immunoblotting for caspase-3 cleavage in oxaliplatin-treated HepG2 cells. Data are mean \pm SD of three independent experiments.

tion induced by oxaliplatin treatment (Ox+ATG7 siRNA vs. Ox+Control siRNA; p<0.05) (Fig. 2e).

In addition to LC3 lipidation, expression of non-conjugated forms of LC3 (LC3-I) was detected using immunoblotting as well. As shown in Fig. 2f, following oxaliplatin treatment, the LC3-I level decreased significantly from $0.36 \pm 0.02$ to $0.32 \pm 0.01$ (control siRNA vs. Ox+siRNA, $\mathrm{p}=0.002$ ). Following oxaliplatin treatment, $A T G 7$ interference induced a significant increase in LC3-I from $0.32 \pm 0.01$ to $0.38 \pm 0.02$ (OX+Control siRNA vs. OX+ATG7 siRNA; p<0.01). However, ATG7 interference treatment alone had almost no inductive effect on the expression of LC3-I (Control siRNA vs. ATG7 siRNA: $0.36 \pm 0.02$ vs. $0.38 \pm 0.01 ; \mathrm{p}=0.115$ ).

Since the increase in LC3 lipidation may either result from the activation of autophagosome formation or from the inhibition of autophagosomal maturation into autolysosomes, CQ, the inhibitor of autophagic-lysosomal protein degradation, was employed for incubation with HepG2 in the presence of oxaliplatin. As shown in Fig. 2g, a highter LC3-II level was detected when CQ was included into the system (Ox+Control siRNA vs. $\mathrm{Ox}+\mathrm{Control}$ siRNA+CQ: $0.35 \pm 0.02$ vs. $0.49 \pm 0.02$; $\mathrm{p}<0.01)$. CQ treatment alone did not influence LC3 lipidation (Control siRNA vs. CQ: $0.18 \pm 0.01$ vs. 0.21 \pm 0.02 ; $\mathrm{p}=0.115$ ).

p62 (sequestosome 1) is a multifunctional signal adaptor that colocalizes with ubiquitinated protein aggregation in many neurodegenerative diseases and proteinopathies of the liver (17). As a link between LC3 and ubiquitinated substrates, p62 can be incorporated into the completed autophagosome and be degraded in autolysosomes (18). Since p62 accumulates when autophagy is inhibited and decreased levels can be observed when autophagy is induced, we used p62 as a marker to further monitor autophagic flux. Western blot analysis (Fig. 2h) revealed that the mean ratio of p62 to GAPDH decreased from $0.50 \pm 0.01$ to $0.20 \pm 0.01$ ( $\mathrm{p}<0.01)$ following oxaliplatin treatment. Thus, increased clearance of p62 protein was confirmed following oxaliplatin treatment.

Altogether, evidenced by the observation of immunofluorescence, quantitative real-time PCR and immunoblotting analyses, our results collectively suggest that autophagy is actively involved in the HepG2 cell response to oxaliplatin treatment, which may be abolished by ATG7 knockdown and CQ treatment. 
Contribution of autophagy to the cell death induced by oxaliplatin. As showed in Fig. 3a, the inhibition of autophagy by $A T G 7$ knockdown significantly amplified the effect of oxaliplatin on cell viability. The combination of oxaliplatin plus ATG7 siRNA produced an almost $13 \%$ decrease compared with the cell viability in the $\mathrm{OX}+\mathrm{Control}$ siRNA group $(46.37 \pm 6.30$ vs. $33.77 \pm 6.10 \%$; $\mathrm{p}=0.024)$. More importantly, $A T G 7$ interference alone had almost no influence on cell viability (100 vs. $89.86 \pm 6.78 \%$; $\mathrm{p}=0.055$ ). After pre-treatment with $\mathrm{CQ}, \mathrm{HepG} 2$ viability was $42.18 \pm 5.83 \%$ after oxaliplatin administration. There was no significant difference in cell viability between ATG7 interference and CQ pre-treatment $(\mathrm{p}=0.095)$. Thus, we confirmed that oxaliplatin-induced cell death was enhanced by the inhibition of autophagy.

Contribution of the inhibition of autophagy to the apoptotic response to oxaliplatin. To determine whether the growth inhibition induced by oxaliplatin treatment did result from apoptotic cell death, flow cytometry with Annexin V-FITC and PI double staining was employed. The results in Fig. 3b showed that oxaliplatin treatment was able to increase the percentage of apoptotic cells from $10.99 \pm 0.88$ to $34.05 \pm 3.10 \%$ $(\mathrm{p}<0.001)$. By detailed analyses, the increased apoptotic effect mainly resulted from the enhanced percentage of cells undergoing early apoptosis (Annexin $\mathrm{V}^{+} / \mathrm{PI}^{-}$). A 48-h treatment with oxaliplatin significantly increased the early apoptosis rate from $16.01 \pm 2.18$ to $4.34 \pm 0.76 \%(\mathrm{p}<0.01)$. Although a slight increase in the late apoptosis and cell death (Annexin $\mathrm{V}^{+} / \mathrm{PI}^{+}$) rate were also noted, no statistical significance was achieved $(18.05 \pm 5.27$ vs. $6.65 \pm 0.13 \%$; $\mathrm{p}=0.064)$.

To further explore the relationship between autophagy and apoptosis in cell death induced by oxaliplatin, autophagy was inhibited by ATG7 interference. Flow cytometric results showed that inhibition of autophagy increased the total fraction of apoptotic cells from $34.05 \pm 3.10$ to $57.17 \pm 2.25 \%$ $(p<0.01)$. Meanwhile, there was no difference in HepG2 cell apoptosis between the ATG7 siRNA group and the Control siRNA group $(12.04 \pm 1.83$ vs. $10.99 \pm 0.88 \%$; $\mathrm{p}=0.542)$.

Since both autophagosome formation and autophagic proteolysis was inhibited by $A T G 7$ knockdown, CQ was further employed to identify the role of autophagic-lysosomal protein degradation in the apoptotic response to oxaliplatin. As shown in Fig. 3c, autophagy inhibition in HepG2 cells by CQ led to a significant increase in the fraction of apoptotic cells from $28.92 \pm 3.64$ to $37.38 \pm 1.83 \%(\mathrm{p}<0.01)$ in the presence of oxaliplatin. CQ treatment alone had almost no influence on the apoptosis rate $(9.48 \pm 1.21$ vs. $11.86 \pm 1.51 \%, \mathrm{p}=0.231)$. These data suggest that inhibition of autophagy by CQ treatment effectively induced HepG2 cell apoptosis following oxaliplatin treatment.

Altogether, our data suggest that the apoptotic cell death induced by oxaliplatin is significantly enhanced by ATG7 interference and CQ treatment as the result of the inhibition of autophagy. Since a $23 \%$ increase in the fraction of apoptotic cells resulted from ATG7 interference was more apparent than the $9 \%$ increase after treatment with $C Q$, we conclude that inhibition of autophagosome formation may function more importantly. Targeting autophagy may increase the sensitivity of HepG 2 cells to oxaliplatin and abrogate the resistance.
Activation of apoptotic pathways by autophagy inhibition in oxaliplatin-treated HepG2 cells. To further confirm the molecular mechanism underlying increased HepG2 cell apoptosis upon the inhibition of autophagy, the change in caspase-3 (apoptosis-related protein) protein level was examined using Western blot analysis. As showed in Fig. 3d, marked caspase-3-specific cleavage was noted following oxaliplatin treatment $(\mathrm{Ox}+\mathrm{Control}$ siRNA vs. Control siRNA: $0.43 \pm 0.02$ vs. $0.28 \pm 0.03 ; \mathrm{p}<0.01)$. Upon inhibition of autophagy by $A T G 7$ knockdown, caspase- 3 cleavage was apparently increased from $0.43 \pm 0.02$ to $0.55 \pm 0.03(\mathrm{Ox}+\mathrm{Control}$ siRNA vs. $\mathrm{Ox}+A T G 7$ siRNA; $\mathrm{p}<0.01)$. The further increase in caspase-3-specific cleavage post $A T G 7$ knockdown supported our hypothesis that autophagy functions importantly during the cell response to oxaliplatin treatment and inhibition of autophagy sensitizes cell death by chemotherapy.

\section{Discussion}

HCC is the third most common cause of death from cancer worldwide. Acting as one of the current treatment modalities, chemotherapy is mainly used to reduce tumor recurrence and prolong survival in unrespectable HCCs. Among the many chemotherapeutic formulas that have been evaluated, oxaliplatin remains one of the most effective agents. Oxaliplatin-based combination chemotherapy has been employed in the treatment of advanced HCC and has shown promising activity in clinical practices (19). Although the anti-proliferation effect of oxaliplatin in HCC cells is due to the induction of apoptosis (20), the resistance to apoptosis as a result of systemic chemotherapeutic treatments has been commonly observed in HCC cells (21).

Autophagy is a major mechanism for degrading longlived cytosolic proteins and the only known pathway for turnover of large cellular structures such as organelles and protein aggregates, the products of which are recycled to generate macromolecules and ATP so as to maintain cellular homeostasis. Therefore, autophagy is a good candidate for a survival mechanism in response to several stresses, such as damaged mitochondria, protein aggregation, pathogens, and nutrient starvation. The stimulation of autophagy in cancer cells was often observed in response to anticancer treatments (22). Resistance of cancers to chemotherapy can be construed as being primarily a resistance to apoptosis commitment. However, it is becoming increasingly evident that some cancer cells mount an autophagic response to stress which delays or suppresses apoptosis (23). Therefore, as an adaptive response to stress, it can be argued that autophagy is first and foremost a survival strategy rather than a death response in some cancer cells. Based on results shown in this report, including increased formation of accumulation of acidic vesicular organelles (Fig. 2b), LC3-positive autophagosomes (Fig. 2c), elevated LC3 lipidation and non-conjugated forms of LC3 (Fig. 2e and f), we concluded that oxaliplatin treatment may induce autophagy in HepG2 cells.

To date, the role and regulation of autophagy in cancer is apparently quite complex and autophagy plays a dual role. At the early stage, autophagy functions as a tumor suppressor, and the autophagic activity is often impaired in cancer cells. In the advanced stage, autophagy seems to promote tumor 
progression. Recent studies have indicated that inhibition of autophagy may improve the chemotherapeutic effect in human breast cancer cells. Cell death was increased by $10 \%$ after inhibition of autophagy (24). In our study, evidenced by dual immunofluorescence observation, LC3 lipidation and LC3-I expression in Western blot analysis, autophagy is actively involved in the HepG2 cell response to oxaliplatin treatment. Further study showed that inhibition of autophagy by ATG7 interference amplified the therapeutic effect of oxaliplatin on HepG2 cells. We then confirmed that inhibition of autophagiclysosomal proteolysis by CQ pre-treatment produced a significant increase in the fraction of apoptotic cells. All of these results indicate that activation of autophagy is closely related to the cell response to oxaliplatin and the chemotherapeutic effect may be enhanced by autophagy inhibition. Therefore, autophagy may play a protective role in response to oxaliplatin treatment in HepG2 cells through the degradation of proteins or organelles that are damaged by anticancer treatments.

Apoptosis and autophagy are genetically regulated, evolutionarily conserved processes that regulate cell fate. The balance between autophagy and apoptosis maintains homeostasis. Many anticancer agents including oxaliplatin exert their effect by stimulating apoptosis (25). HCCs with poor prognosis are characterized by rapid cell proliferation and strong expression of anti-apoptotic genes (26). Recent studies have found that inhibition of autophagy may cause abnormal proteins and organelles to accumulate, thereby promoting apoptosis or necrosis. In human prostate cancer PC-3 cells, the apoptosis induced by sulforaphane increased after inhibition of autophagy (27). Similar findings were also observed in colon cancer cells; one study found that 5-FU-induced apoptosis in colon cancer cells was enhanced by the inhibitor of autophagy (28). These results reveal that autophagy may play an important role in cell death decisions and can protect cells by preventing them from undergoing apoptosis. In the present study, the percentage of apoptotic cells increased significantly after inhibition of autophagy by $A T G 7$ knockdown and CQ pre-treatment. Along with apoptotic cell death, the cleavage of caspase-3 increased consistently, which further confirmed the enhanced apoptosis following autophagy inhibition. Therefore, our results suggest that inhibition of autophagy sensitizes HCC cells to oxaliplatin chemotherapy by enhancing the rate of apoptotic cell death or by converting the autophagic process to an apoptotic process.

p62/SQSTM1 is commonly found in protein aggregation diseases affecting the liver. Liver-specific autophagy defects in mice cause accumulation of p62 aggregates (29). Recent findings link p62 activity to the extrinsic apoptosis pathway, and suggest that the modulation of $p 62$ by autophagy is a key factor in carcinogenesis (30). Our results showed that autophagy-mediated clearance of p62 protein after oxaliplatin treatment has an essential role in the protection of cancer cells against chemotherapy. However, considering the controversial role of autophagy in cell death, the time spot and target site of autophagy in oxaliplatin-inhibited HepG2 are still needed to be further explored.

In summary, autophagy may play an important role in chemoresistance to oxaliplatin in HCC cells, and inhibition of autophagy may improve the chemotherapeutic effect of oxaliplatin in HepG2 cells through the activation of caspase-3 cleavage. Therefore, pharmacological inhibition of autophagy may be a promising strategy for the adjuvant chemotherapy of $\mathrm{HCC}$.

\section{Acknowledgements}

This study was supported by the Nature Science Foundation of China (30872511), Shanghai Science and Technology Commission Grant (08QA14047, 10ZR1419400) and Shanghai Charity Foundation for Cancer Research. We are very grateful to Dr Ruyuan Zhang and Guangtao Sun for the discussion during the research.

\section{References}

1. Caldwell S and Park SH: The epidemiology of hepatocellular cancer: from the perspectives of public health problem to tumor biology. J Gastroenterol 19: 96-101, 2009.

2. McGlynn KA and London WT: Epidemiology and natural history of hepatocellular carcinoma. Best Pract Res Clin Gastroenterol 19: 3-23, 2005.

3. Kudo M: Hepatocellular carcinoma 2009 and beyond: from the surveillance to molecular targeted therapy. Oncology 75: 1-12, 2008.

4. Lee JG, Choi SB, Kim KS, Choi JS, Lee WJ and Kim BR: Central bisectionectomy for centrally located hepatocellular carcinoma. Br J Surg 95: 990-995, 2008.

5. Roberts LR: Sorafenib in liver cancer - just the beginning. $N$ Engl J Med 359: 420-422, 2008.

6. Misset JL, Bleiberg H, Sutherland W, Bekradda M and Cvitkovic E: Oxaliplatin clinical activity: a review. Crit Rev Oncol Hematol 35: 75-93, 2000

7. Louaf S, Boige V, Ducreux M, et al: Gemcitabine plus oxaliplatin (GEMOX) in patients with advanced hepatocellular carcinoma (HCC): results of a phase II study. Cancer 109: 1384-1390, 2007.

8. Tsuchihara K, Fujii S and Esumi H: Autophagy and cancer: dynamism of the metabolism of tumor cells and tissues. Cancer Lett 278: 130-138, 2009.

9. Apel A, Zentgraf H, Büchler MW and Herr I: Autophagy-A double-edged sword in oncology. Int J Cancer 125: 991-995, 2009.

10. Yousefi S and Simon HU: Autophagy in cancer and chemotherapy. Results Probl Cell Differ 49: 183-190, 2009.

11. Qiu W, David D, Zhou B, et al: Down-regulation of growth arrest DNA damage-inducible gene 45beta expression is associated with human hepatocellular carcinoma. Am J Pathol 162: 1961-1974, 2003.

12. Shvets E and Elazar Z: Autophagy-independent incorporation of GFP-LC3 into protein aggregates is dependent on its interaction with p62/SQSTM1. Autophagy 16: 1054-1056, 2008.

13. Kabeya Y, Mizushima $\mathrm{N}$ and Ueno T: LC3, a mammalian homologue of yeast Apg8p, is localized in autophagosome membranes after processing. EMBO J 19: 5720-5728, 2000.

14. Komatsu M, Waguri S, Ueno T, et al: Impairment of starvationinduced and constitutive autophagy in ATG7-deficient mice. J Cell Biol 169: 425-434, 2005.

15. Qiu W, Zhou B, Zou H, et al: Hypermethylation of growth arrest DNA damage-inducible gene 45 beta promoter in human hepatocellular carcinoma. Am J Pathol 165: 1689-1699, 2004.

16. Karim MR, Kanazawa T, Daigaku Y, Fujimura S, Miotto G and Kadowaki M: Cytosolic LC3 ratio as a sensitive index of macroautophagy in isolated rat hepatocytes and H4-II-E cells. Autophagy 3: 553-560, 2007.

17. Olivé M, van Leeuwen FW, Janué A, Moreno D, TorrejónEscribano B and Ferrer I: Expression of mutant ubiquitin $(\mathrm{UBB}+1)$ and $\mathrm{p} 62$ in myotilinopathies and desminopathies. Neuropathol Appl Neurobiol 34: 76-87, 2008.

18. Bjørkøy G, Lamark T, Brech A, et al: p62/SQSTM1 forms protein aggregates degraded by autophagy and has a protective effect on Huntingtin-induced cell death. J Cell Biol 171: 603-614, 2005.

19. Boige V, Raoul JL, Pignon JP, et al: Multicentre phase II trial of capecitabine plus oxaliplatin (XELOX) in patients with advanced hepatocellular carcinoma: FFCD 03-03 trial. Br J Cancer 97: 862-867, 2007. 
20. Wang Z, Zhou J, Fan J, et al: Oxaliplatin induces apoptosis in hepatocellular carcinoma cells and inhibits tumor growth. Expert Opin Investig Drugs 18: 1595-1604, 2009.

21. Bruix J, Hessheimer AJ, Forner A, Boix L, Vilana R and Llovet JM: New aspects of diagnosis and therapy of hepatocellular carcinoma. Oncogene 25: 3848-3856, 2006.

22. Ding WX, Ni HM, Gao W, et al: Differential effects of endoplasmic reticulum stress-induced autophagy on cell survival. J Biol Chem 282: 4702-4710, 2007.

23. Ogier-Denis E and Codogno P: Autophagy: a barrier or an adaptive response to cancer. Biochim Biophys Acta 1603: 113-128, 2003

24. Abedin MJ, Wang D, McDonnell MA, Lehmann U and Kelekar A: Autophagy delays apoptotic cell death in breast cancer cells following DNA damage. Cell Death Differ 22: 500-510, 2006.

25. Arango D, Wilson AJ, Shi Q, et al: Molecular mechanisms of action and prediction of response to oxaliplatin in colorectal cancer cells. Br J Cancer 91: 1931-1946, 2004.
26. Lee JS, Chu IS, Heo J, et al: Classification and prediction of survival in hepatocellular carcinoma by gene expression profling. Hepatology 40: 667-676, 2004.

27. Herman-Antosiewicz A, Johnson DE and Singh SV: Sulforaphane causes autophagy to inhibit release of cytochrome $\mathrm{c}$ and apoptosis in human prostate cancer cells. Cancer Res 66: 5828-5835, 2006.

28 Li J, Hou N, Faried A, Tsutsumi S, Takeuchi T and Kuwano H: Inhibition of autophagy by 3-MA enhances the effect of 5-FU-induced apoptosis in colon cancer cells. Ann Surg Oncol 16: 761-771, 2009.

29. Komatsu M, Waguri S, Koike M, et al: Homeostatic levels of p62 control cytoplasmic inclusion body formation in autophagydeficient mice. Cell 131: 1149-1163, 2007.

30. Mathew R, Karp CM, Beaudoin B, et al: Autophagy suppresses tumorigenesis through elimination of p62. Cell 137: 1062-1075, 2009. 